\title{
DECELERATION AND HEATING CONSTRAINED FOOTPRINT OF SHUTTLE VEHICLES $\dagger$
}

\author{
Jeng-Shing Chern and Ching-Yew YANG \\ Chung Shan Institute of Science and Technology, Lungtan, Taiwan 325. Republic of China
}

NGUYEN X. VINH

Department of Acrospace Engineering, University of Michigan, Ann Arbor, MI 48109-2140. U.S.A.

and

JOHN M. HANSON

Analytic Services, Inc., Arlington, VA, U.S.A

(Received 14 February 1985)

\begin{abstract}
With increasing frequency in shuttle operation, it is of interest to have more than one or two landing fields within the boundary of the reachable area of the reentry vehicle. This boundary, called the footprint, depends on the aerodynamic characteristics of the vehicle and is severely restricted by the deceleration and heating constraints imposed upon the atmospheric reentry trajectory. This paper gives a general assessment of the footprint as a function of various deceleration and heating constraints. The difficulties in the computation of the three-dimensional reentry trajectories with optimal modulation in both the angle-of-attack and the bank angle are alleviated by the following devices: (a) nondimensionalizing of the equations of motion and use of the density as the altitude variable; (b) use of the classical integrals of the motion; (c) transformation of the adjoint variables into physical variables; and (d) spherical rotation of the coordinates.
\end{abstract}

\section{INTRODUCTION}

The lateral maneuver of a lifting reentry vehicle, exemplified by the space shuttle entry, is severely restricted by deceleration and heating constraints. By nature, the computation of optimal trajectories is very sensitive. Until now only purely numerical programs for specific vehicles have led to some meaningful results which are, however, limited in scope[1,2]. This paper gives a general assessment of the footprint as a function of various deceleration and heating constraints. The difficulties in the computation of the three-dimensional reentry trajectories with optimal modulation in both the angle-of-attack and the bank angle are alleviated by the following devices: (a) nondimensionalizing of the equations of motion and use of the density as the altitude variable; (b) use of the classical integrals of the motion; (c) transformation of the adjoint variables into physical variables; and (d) spherical rotation of the coordinates.

With the dimensionless equations of motion, it is possible to specify just the maximum lift-to-drag ratio ( $L /$ $D)_{\max }$ as the sole physical characteristic of the vehicle. Hence the analysis applies to a whole class of vehicles having the same value for $(L / D)_{\max }$. The integrals of the motion allow for the reduction of the order of the canonical system. The transformation of the adjoint variables into physical variables makes the initial guess of unknown constants a much less arbitrary task. Finally, the rotation of the coordinates on a spherical earth reduces the com-

\footnotetext{
† Paper presented at the 35th Congress of the International Astronautical Federation. Lausanne. Switzerland, 8-13 October 1984.
}

putation of the footprint to a swept operation through a scanning parameter. The new technique removes the restriction of equilibrium glide reported previously in the literature [3, 4]. The exact optimal three-dimensional trajectory for maximum lateral range is found by the use of a direct shooting method. Then, the optimal trajectory for the same purpose but obtained in a reduced phase coordinate system using the equilibrium glide assumption is compared with the exact optimal trajectory. Finally, the footprints with and without deceleration and heating constraints are constructed to show the effect of the constraints on the size of the footprint. Several values of deceleration and thermal constraints $|5|$ are imposed on the optimal trajectories leading to the footprints. The equilibrium glide assumption is again applied in order to reduce the numerical computation load.

\section{DECELERATION AND HEATING CONSTRAINTS}

During entry, the deceleration due to the aerodynamic force with coefficient $C$ is

$$
a=-\frac{d V}{d t}=\frac{\rho S V^{2} C}{2 m}
$$

At the beginning of the entry the density $\rho$ is negligibly small and hence $a$ is small. As the density increases, the deceleration becomes larger. Near the end of the flight, the speed decreases to a very small value and again the deceleration is small. As a consequence, $a$ passes through at least one maximum value, and for the comfort of the crew and passengers, this peak deceleration must be controlled to an acceptable level. 
Thermal control is another factor of prime consideration. During entry, the kinetic energy. $1 / 2 V^{2}$. decreases from a high value to nearly zero. A fraction of this energy is converted into heat and absorbed by the vehicle. Sevcral texts $[6,7]$ discuss the important aspects of heat flow into reentry vehicles. Here we shall consider only the maximum temperature on a selected point of the vehicle. To control the temperature, it suffices to control the heating rate at that point. According to Lees $[8]$, the heating rate at any point on a body is a fraction

$$
f=q / q
$$

of the heating rate $q_{s}$ at a stagnation point of radius of curvature $R$

$$
q_{1}=\frac{\kappa}{\sqrt{R}}\left(\frac{\rho}{\rho_{n}}\right)^{\prime \prime}\left(\frac{V}{V_{n}}\right)^{\prime \prime \prime}
$$

where $\kappa$ is a constant, and $\rho_{i}$ and $V_{i}$ are the reference density and reference speed, respectively. The constant exponents $n$ and $m$ depend on the type of boundary-layer flow. For laminar flow, we have $n=1 / 2$. Under the assumptions that the viscosity coefficient varies as the square root of the absolute temperature and that the flow between the bow shock wave and the stagnation point is incompressible, we can use the value $m=3$.

\section{DIMENSIONLESS EQUATIONS OF MOTION}

The motion of the vehicle. considered as a mass point, is defined by the six variables $r, \theta, \phi, V, \gamma$ and $\psi$ as shown in Fig. 1. Using a parabolic drag polar of the form

$$
C_{1}=C_{(1)}+K C_{l}^{1} \text {, }
$$

we define the normalized lift coefficient

$$
\lambda=C_{l} / C_{l}^{*}
$$

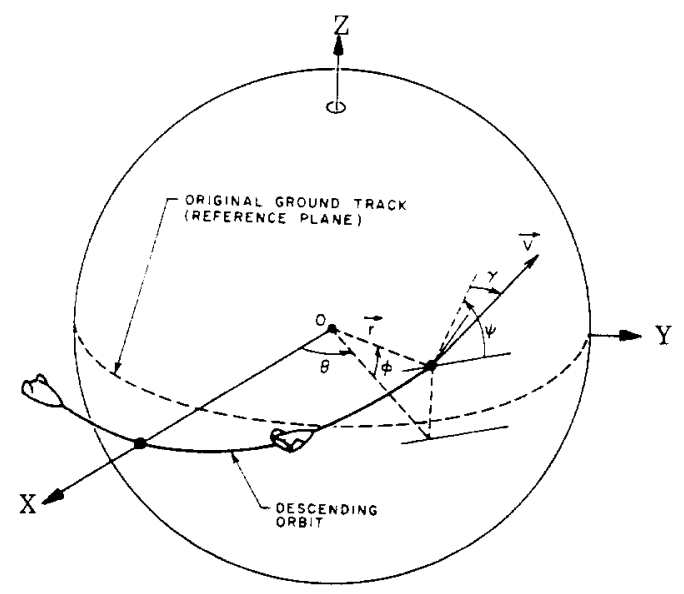

Fig. 1. Coordinate system and state variables: the longitude $\theta$. the latitude $\phi$, the position vector $\vec{r}$, the heading angle $\psi$. the tlight path angle $\gamma$, and the velocity vector $\vec{v}$. where $C_{L}^{*}$ is the lift coefficient corresponding to the maximum lift-to-drag ratio, $E^{*}$. With given values of $C_{b 0}$ and $K$ assumed constant at hypersonic speed, we easily compute

$$
C_{i}^{*}=\sqrt{\frac{C_{D 0}}{K}}, \quad C_{n}^{*}=2 C_{m \infty}, \quad E^{*}=\frac{1}{2 \sqrt{C_{m 0} K}} .
$$

The earth is assumed at rest and with a locally exponential atmosphere

$$
\frac{d \rho}{\rho}=-\beta d r
$$

where $\rho$ is the atmospheric density and $\beta$, the reciprocal of the scale height, is a function of the radial distance $r$. Its gravitational force field is the usual inverse-square force field

$$
g=\frac{\mu}{r^{2}}
$$

where $\mu$ is the gravitational constant. Without loss of generality, we can use the equatorial plane as the reference plane. By introducing the following dimensionless variables:

$$
Z=\frac{\rho S C_{L}^{*}}{2 m} \sqrt{\frac{r}{\beta}}, \quad v=\frac{V^{2}}{g r} . \quad s=\int_{0}^{t} \frac{V \cos \gamma}{r} d t
$$

we have the dimensionless equations of motion[4]

$$
\begin{aligned}
& \frac{d Z}{d s}=-k^{2} Z \tan \gamma \\
& \frac{d v}{d s}=-\frac{k Z v\left(1+\lambda^{2}\right)}{E^{*} \cos \gamma}-(2-v) \tan \gamma \\
& \frac{d \gamma}{d s}=\frac{k Z \lambda \cos \sigma}{\cos \gamma}+1-\frac{1}{v} \\
& \frac{d \theta}{d s}=\frac{\cos \psi}{\cos \phi} \\
& \frac{d \phi}{d s}=\sin \psi \\
& \frac{d \psi}{d s}=\frac{k Z \lambda \sin \sigma}{\cos ^{2} \gamma}-\cos \psi \tan \phi .
\end{aligned}
$$

The aerodynamic controls are in the form of the bank angle, $\sigma$, and the normalized lift coefficient, $\lambda$. Hence when $\lambda=1$ the flight is at maximum lift-to-drag ratio. The dimensionless variable $Z$ replaces the altitude and the dimensionless kinetic energy $v$ is a measure of the speed. They are the modified Chapman's variables. $s$ is the dimensionless arc length.

The equations in (10) contain only two constant parameters which need to be specified. The first parameter. $k^{2}=\beta r$, is a characteristic of the atmosphere. For the computation, we select the value $k^{2}=900$ for the earth. The second parameter. $E^{*}$, is the maximum lift-to-drag 
ratio and is a performance characteristic of the vehicle. For the present numerical example we shall take the value $E^{*}=2$.

From eqn (1) and with the definition (9), the dimensionless deceleration corresponding to an aerodynamic coefficient $C_{L}=C_{L}^{*}$ is

$$
\bar{a}=\frac{a}{g}=k Z v
$$

On the other hand, from eqn (3) we can construct the function

$$
\bar{q}=\sqrt{k} Z^{1 / 2} v^{3 / 2}
$$

representing the dimensionless heating rate.

\section{VARIATIONAL FORMULATION}

The footprint of a reentry vehicle is defined as the curve limiting the reachable domain on the surface of the earth when the reentry point is specified. The two control variables in (10) are subject to the constraints

$$
|\lambda| \leq \lambda_{\max }, \quad|\sigma| \leq \sigma_{\max }
$$

These two controls are to be selected to bring the vehicle from a certain prescribed initial condition to a certain partially prescribed final condition, such that the cross range is maximized for each prescribed final longitudinal range $\theta_{f}$. The footprint can then be constructed.

Using the maximum principle, we introduce the adjoint vector $\vec{p}$ to form the Hamiltonian

$$
\begin{aligned}
H & =-k^{2} Z p_{Z} \tan \gamma-p \\
& \times\left[\frac{k Z v\left(1+\lambda^{2}\right)}{E^{*} \cos \gamma}+(2-v) \tan \gamma\right] \\
& +p_{\gamma}\left[\frac{k Z \lambda \cos \sigma}{\cos \gamma}+\left(1-\frac{1}{v}\right)\right] \\
& +p_{H}\left[\frac{\cos \psi}{\cos \phi}\right]+p_{\phi} \sin \psi+p_{\downarrow} \\
& \times\left[\frac{k Z \lambda \sin \sigma}{\cos ^{2} \gamma}-\cos \psi \tan \phi\right] .
\end{aligned}
$$

The components of $\vec{p}$ are governed by the adjoint equations

$$
\frac{d p_{i}}{d s}=-\frac{\partial H}{\partial x}, \quad x=Z, v, \gamma, \theta, \phi, \psi
$$

It has been shown that the system has four integrals

$$
\begin{aligned}
& H=C_{0} \\
& p_{H}=C_{1} \\
& p_{\phi}=C_{2} \sin \theta-C_{3} \cos \theta \\
& p_{4}=C_{1} \sin \phi+\left(C_{2} \cos \theta+C_{3} \sin \theta\right) \cos \phi
\end{aligned}
$$

where $C_{1}, C_{1}, C_{2}$, and $C_{3}$ are constants of integration. In order to maximize the cross range $\phi_{f}$ for each prescribed $\theta_{f}$, we do not specify the arc length and the final heading $\psi_{f}$. Hence we have the transversality conditions

$$
C_{i s}=0, \quad p_{v s_{i}}=0, \quad p_{\mathrm{b}_{i}}=1
$$

For the remaining three adjoint variables $p_{\gamma}, p_{\gamma}$ and $p_{\gamma}$. we define the following three new adjoint variables

$$
P=k^{2} Z p_{\gamma}, \quad N=v p_{r}, \quad Q=p_{\gamma} \cos \gamma
$$

In terms of these variables, the Hamiltonian integral becomes

$$
\begin{aligned}
H & =-\frac{k N Z\left(1+\lambda^{2}\right)}{E^{*} \cos \gamma}+\frac{k Z \lambda}{\cos ^{2} \gamma} \\
& \times\left(Q \cos \sigma+p_{w} \sin \sigma\right) \\
& -\frac{Q(1-v)}{v \cos \gamma}-\frac{[v P+(2-v) N]}{v} \\
& \times \tan \gamma+H_{1}=0
\end{aligned}
$$

where

$$
\begin{aligned}
H_{1} & =C_{1} \cos \phi \cos \psi-C_{2} \\
& \times(\sin \phi \cos \theta \cos \psi-\sin \theta \sin \psi) \\
& -C_{3}(\sin \phi \sin \theta \cos \psi+\cos \theta \sin \psi),
\end{aligned}
$$

a function of the state variables. The new adjoint variables $P, N$ and $Q$ are governed by the system of differential equations

$$
\begin{aligned}
\frac{d P}{d s} & =-k^{2}\left\{-H_{1}+\frac{Q(1-v)}{v \cos \gamma}\right. \\
& \left.+\frac{[v P+(2-v) N]}{v} \tan \gamma\right\} \\
\frac{d N}{d s} & =-\frac{2 N}{v} \tan \gamma-\frac{Q}{v \cos \gamma} \\
\frac{d Q}{d s} & =2 H_{1} \sin \gamma-\frac{Q(1-v)}{v} \tan \gamma \\
& +\frac{\left(1-2 \sin ^{2} \gamma\right)}{v \cos \gamma}[v P+(2-v) N] \\
& -\frac{k N Z\left(1+\lambda^{2}\right)}{E^{*}} \tan \gamma .
\end{aligned}
$$

The optimal solution to the problem is governed by eqns (10) for the state variables, and eqns (21) for the adjoint variables. The integration requires six parameters $C_{1}, C_{2}$, $C_{3}, P_{0}, N_{0}$, and $Q_{0}$ to satisfy the final and transversality conditions, where $P_{0}, N_{0}$, and $Q_{0}$ are the initial values of $P, N$, and $Q$, respectively. The Hamiltonian integral, eqn (19), can be used to eliminate one of the parameters. During the integration, whenever interior lift and bank 
controls are used, we have

$$
\begin{aligned}
\lambda \cos \sigma & =\frac{E^{*} Q}{2 N \cos \gamma}, \lambda \sin \sigma=\frac{E^{*} p_{0}}{2 N \cos \gamma .} \\
\lambda^{2} & =\frac{E^{* 2}\left(Q^{2}+p_{i}^{2}\right)}{4 N^{2} \cos ^{2} \gamma} .
\end{aligned}
$$

For maximum bank angle, $\sigma=\sigma_{\text {mat }}$, with variable lift coefficient, the normalized lift control is $[4]$

$$
\lambda=\frac{E^{*}}{2 N \cos \gamma}\left(Q \cos \sigma_{m, a,}+p_{b} \sin \sigma_{\mathrm{m} w,}\right) .
$$

On the other hand, the variable bank angle used with a maximum lift coefficient, $\lambda=\lambda_{\operatorname{man}}$. is obtained from

$$
\tan \sigma=\frac{P_{\mathrm{N}}}{Q} .
$$

\section{OPTIMAL TRAJECTORIES FOR MAXIMUM CROSS RANGE.}

\subsection{Exact solution}

In the problem of maximum cross range, it is proposed to find the lift and bank modulation to maximize the final latitude $\phi_{,}$while the final longitudinal range $\theta_{i}$ is free. For the numerical computation, we use the initial condition

$\left(Z_{11}, v_{0}, \gamma_{11}, \theta_{0}, \phi_{0}, \psi_{01}\right)$

$$
=\left(0.0005,1 .,-4^{\circ}, 0,0,0\right)
$$

with the final condition at free $s$,

$\left(Z_{i}, v_{f}, \gamma_{i}, \theta_{i}, \phi_{i}, \psi_{i}\right)$

$=(30 ., 0.001$, free. free, max., free $)$.

Then we have two more transversality conditions besides eqns (17), which are

$$
C_{1}=0, \quad p_{\gamma_{j}}=0
$$

$p_{\mathrm{d}}$ and $p_{\mathrm{u}}$ can be determined by using eqns (16), (17), and (27),

$$
\begin{aligned}
& p_{\mathrm{b}}=\cos \left(\theta_{f}-\theta\right) \\
& p_{\omega}=\cos \phi \sin \left(\theta_{f}-\theta\right) .
\end{aligned}
$$

Also, by the definition of $Q$ in eqns (18), the transversality condition $p_{\gamma_{t}}=0$ in eqns $(27)$ becomes the condition

$$
Q_{i}=0
$$

The Hamiltonian integral remains the same as in eqn (19) and the system of differential equations for $P, N$, and $Q$ is the same as in eqns (21). except that now $H_{1}$ takes the form

$$
H_{1}=\sin \psi \cos \left(\theta_{1}-\theta\right)-\sin \phi \cos \psi \sin (\theta,-\theta) .
$$

In summary, the optimal trajectory is obtained by integrating the state equations. eqns (10), and the adjoint equations, eqns $(21)$, with $H_{1}$ given in eqn $(30)$ ). from the initial condition, using the optimal control laws of eqns (22), (23) and (24). This will require selecting three arbitrary parameters: the final value $\theta_{f}$. and two of the three initial values $P O, N o$, and $Q O$. One of these three values can be obtained from the Hamiltonian integral. eqn (19), with $H_{1}$ given in eqn (30). At $\theta=\theta_{1}$. the conditions of $Z_{f}, v$ and $Q_{i}$ are used to adjust the values of the guessed parameters.

In order to make the initial guess of unknown parameters a much less arbitrary task, we will change the parameters describing the adjoint variables, $N_{w}$ and $Q_{\ldots,}$ to physical parameters. From the first two equations of (22). since $\theta_{1,}=\phi_{0}=0$ we have $p_{t_{1} .}-\sin \theta_{t}$ and

$$
N_{0,}=\frac{E^{*} \sin \theta_{f}}{2 \lambda, \sin \sigma_{0} \cos \gamma_{n}} . \quad Q_{n}=\frac{\sin \theta_{f}}{\tan \sigma}
$$

where $\lambda_{0}$ and $\sigma_{i}$ are the initial values of the normalized lift coefficient and bank angle, respectively. Therefore, the initial guess is equivalent to making an initial guess on the new set of parameters $\left(\theta_{j}, \lambda_{m,} \sigma_{c}\right)$. All parameters in the new set with physical meaning and this provides us with a much easier assessment of their values. The optimal trajectory for maximum cross range is solved with the set of values $\left(\theta_{i}, \lambda_{i}, \sigma_{i}\right)=(1.09452,1.05331$ $\left.59.9498^{\circ}\right)$. Figure 2 presents the optimal trajectory and the variations of the state variables and control variables. It can be seen that the flight path angle oscillates about and stays near the value of $0^{\circ}$. and that the normalized lift coefficient oscillates about and stays near the value of 1.0. except near the end of the trajectory where substantial lift maneuver is made so that the final conditions can be met.

\subsection{Equilibritum glide solution}

The so-called equilibrium glide condition consists of assuming that the glide angle is small and stays nearly constant. From Fig. 2 it is seen that in the exact optimal trajectory the glide angle oscillates about and stays near the value of $0^{\circ}$. Hence for the equilibrium glide condition we make the assumption that

$$
\gamma \approx 0, \frac{d \gamma}{d s} \approx 0
$$

With this, the equation for $\gamma$ becomes the cquilibrium equation

$$
k Z=\frac{1-v}{i \cdot \lambda \cos \sigma} .
$$




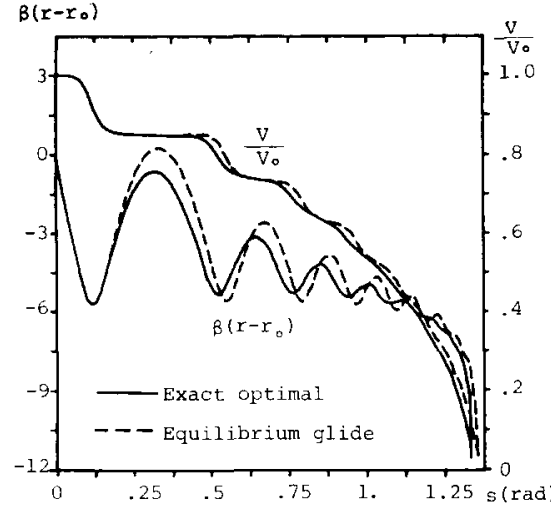

(a)

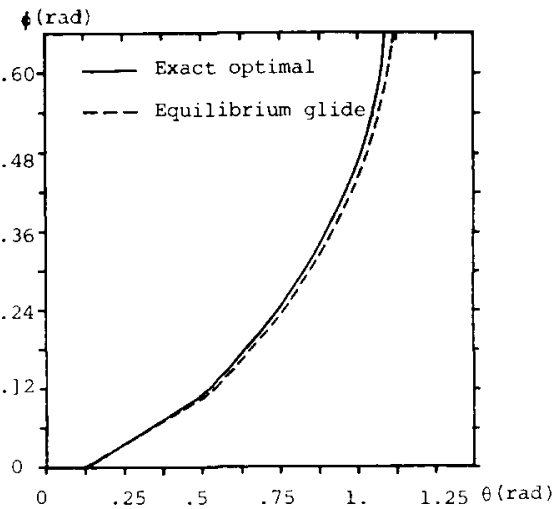

(c)

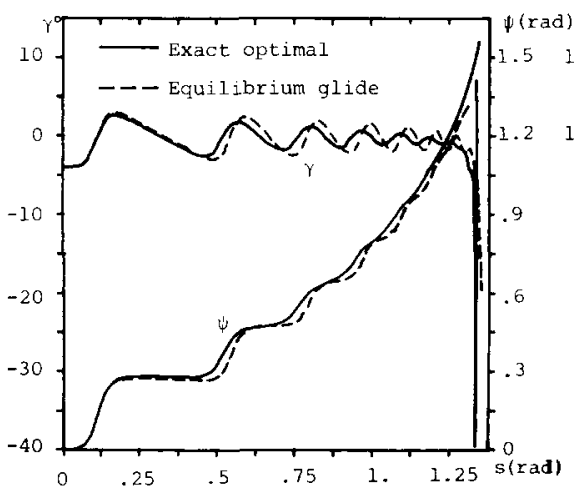

(b)

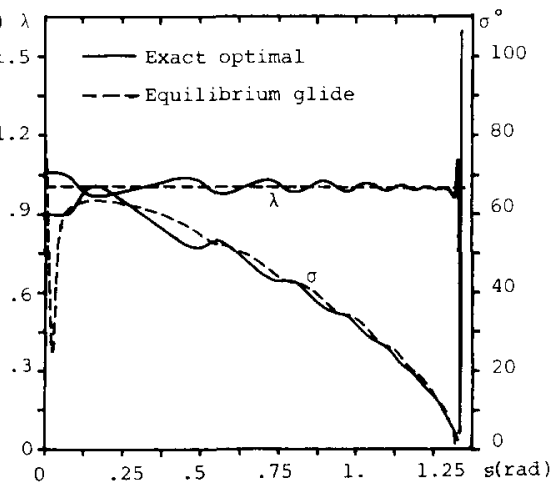

(d)

Fig. 2. Optimal trajectories for maximum cross range. (a) Dimensionless altitude $\beta\left(r-r_{o}\right.$ ) and velocity ratio $V / V$, vs arc length $s$. (b) Flight path angle $\gamma$ and heading angle $\psi$ vs arc length. (c) Latitudinal range $\phi$ vs longitudinal range $\theta$. (d) Normalized lift coefficient $\lambda$ and bank angle $\sigma$ vs arc length.

This equation is used to evaluate the altitude variable $Z$. With the equation for $Z$ eliminated, we have the following reduced set of state equations

$$
\begin{aligned}
& \frac{d v}{d s}=-\frac{\left(1+\lambda^{2}\right)(1-v)}{E^{*} \lambda \cos \sigma} \\
& \frac{d \theta}{d s}=\frac{\cos \psi}{\cos \phi} \\
& \frac{d \phi}{d s}=\sin \psi \\
& \frac{d \psi}{d s}=\frac{(1-v)}{v} \tan \sigma-\cos \psi \tan \phi .
\end{aligned}
$$

It is easy to verify that in this reduced set of state equations, we preserve the classical integrals of eqns (16). Also, we have the transversality conditions $C_{0}=C_{1}=$ $p_{\psi_{f}}=0$ and $p_{\phi_{j}}=1$, and the solutions for $p_{\phi}$ and $p_{\psi}$ given in (28). It has been shown $[4,5]$ that for lift control the optimal equilibrium glide is effected at maximum liftto-drag ratio, namely $\lambda=1$, and for bank control we either have $|\sigma|=\sigma_{\text {max }}$ or a variable bank angle such that

$$
\begin{aligned}
& \tan \sigma=\frac{(1-v)}{v} \\
& \times \frac{\cos \phi \sin \left(\theta_{f}-\theta\right)}{\cos \left(\theta_{f}-\theta\right) \sin \psi-\cos \psi \sin \phi \sin \left(\theta_{f}-\theta\right)} .
\end{aligned}
$$

The problem is thus reduced to a one-parameter problem in the value $\theta_{f}$. In this formulation, the condition on $Z$, is no longer enforced and we use the prescribed final value $v_{f}=0.001$ to adjust the sole parameter $\theta_{f}$.

For numerical computation, we use the control law (35) with $\lambda=1$ to integrate the full set of exact state equations (10). The initial state used is (25) except that the initial value $v_{c \prime}$ is 0.999 instead of 1.0 . The purpose of this change is to give a defined value for $\sigma$ at the initial time. The maximum value of the bank angle $\sigma_{\text {mix }}$ is selected to be $85^{\circ}$. The results are also presented in Fig. 2 in dashed lines for the purpose of comparison with the exact results.

From Fig. 2 it is easy to see that the optimal solution under the equilibrium glide condition is very close to the exact optimal solution. It results in a slightly smaller 
value of $\phi_{\text {, }}$ and a slightly larger value of $\theta_{,}$. Quantitatively, we have $\left(\theta_{f}, \phi_{f}\right)=(1.0945,0.6577)$ for the exact solution and $\left(\theta_{i}, \phi_{j}\right)=(1.1229,0.6575)$ for the equilibrium glide solution. Hence we will say that the equilibrium glide solution is a very good suboptimal solution and we shall use the equilibrium glide condition in obtaining the solution of the footprint, either without or with deceleration and heating constraints.

\section{THE FOOTPRINT WITHOUT CONSTRAINT}

\subsection{Exact formulation}

If the reentry vehicle is initially in a close circular orbit and the position for leaving the orbit is not prescribed. then the reachable domain on the surface of the earth will be a zone between the latitudes $-\phi_{\max }$ and $\phi_{\max }$. The footprint of a reentry vehicle is defined as the curve limiting the reachable domain on the surface of the earth if the reentry point is specified. To construct the footprint we have to find the maximum cross range for each prescribed final longitudinal range $\theta_{,}$. As the final longitudinal range is no longer free, $p_{\theta}=C_{1} \neq 0$. We can normalize all the adjoint variables by taking $C_{1}=1$. This is the same as reducing proportionally all the adjoint variables by the factor $1 / C_{1}$ and using the new constants $k_{2}=C_{2} / C_{1}$ and $k_{3}=C_{3} / C_{1}$. Then for $p_{0}$ and $p_{1}$ we have the solution

$$
\begin{aligned}
& p_{1}=k_{2} \sin \theta-k_{3} \cos \theta \\
& p_{1}=\sin \phi+\left(k_{2} \cos \theta+k_{3} \sin \theta\right) \cos \phi .
\end{aligned}
$$

With $C_{10}=0$, the Hamiltonian integral is given by eqn (19) but with

$$
\begin{aligned}
H_{1} & =\cos \phi \cos \psi-k_{2} \\
& \times(\sin \phi \cos \theta \cos \psi-\sin \theta \sin \psi) \\
& -k_{3}(\sin \phi \sin \theta \cos \psi+\cos \theta \sin \psi) .
\end{aligned}
$$

The lift and bank controls, $\lambda$ and $\sigma$, and the adjoint equations for $P . N$ and $Q$ are the same as given in eqns (19)-(24) except that $H_{1}$ in eqn (20) must be replaced by $H_{1}$ as given in eqn (37). The problem becomes a fourparameter problem in terms of the constants $k_{2}, k_{3}$ and two of the three initial values $P_{10}, N_{0}$ and $Q_{o}$ (since onc of them can be obtained by the Hamiltonian integral). To generate the footprint, we can use one parameter as a scanning parameter. Hence it is a three-parameter problem.

As before, we can change these parameters to the new parameters with physical meaning. This change can be accomplished through the use of a rotation of coordinates $[4,9]$. Consider a trajectory leading from the initial point $M_{c}$, to the final point $M_{f}$ on the footprint $C$ as shown in Fig. 3. Let $M_{t} \theta \phi$ be the original coordinate system and let $M_{i} \theta^{\prime} \phi^{\prime}$ be the rotated coordinate system. The $M_{v}, \theta^{\prime}$ axis is along a great circle parallel to the tangent to the footprint at $M_{f}$ and the $M_{o} \phi^{\prime}$ axis is along the great circle orthogonal to the great circle $M_{t} \theta^{\prime}$. This rotation introduces the new heading angle $\psi_{0}^{\prime} \neq 0$, but this angle can be used as the scanning parameter. In this rotated axis system the point $M$, is obtained by maximizing $\phi$, with free $\theta_{1}^{\prime}$. Hence the formulation in Section 5.1 with the prime notation on $\theta, \phi$, and $\psi$ applies. The unknown set of parameters to be found is now $\left(\theta_{1}^{\prime}, \lambda_{1}, \sigma_{*}\right)$. For cach value of the scanning parameter $\psi^{\prime}$, from $-90^{\circ}$ to $+90^{\circ}$. We solve the maximum cross range problem to obtain the final point $\left(\theta_{f}^{\prime}, \phi_{f}^{\prime}\right)$. Using a formula in spherical trigonometry, it can be shown that the position in the original coordinate system is obtained from

$$
\begin{aligned}
& \tan \theta_{t}=\tan \theta_{t}^{\prime} \cos \psi_{t}^{\prime}+\frac{\tan \phi_{i}^{\prime} \sin \psi_{t}^{\prime}}{\cos \theta_{t}^{\prime}} \\
& \sin \phi_{t}=\sin \phi_{t}^{\prime} \cos \psi_{t}^{\prime}-\sin \theta_{t}^{\prime} \cos \phi_{t}^{\prime} \sin \psi_{m}^{\prime}
\end{aligned}
$$

\subsection{Equilibrium glide solution}

Since it is very tedious to use the exact formulation to generate the footprint, and since the equilibrium glide solution is a very good suboptimal solution, we shall use this assumption of equilibrium glide as a reliable and efficient tool for solving this complex problem.

From the reduced set of state equations (34) we deduce that $\lambda=1$. The bank angle is either $\sigma=\sigma_{\text {nili }}$ or is an interior bank angle as given by $|4|$

$\tan \theta=\frac{1}{H_{1}}\left(\frac{1-V}{r}\right)$

$$
\times\left|\sin \phi+\left(k_{2} \cos \theta+k_{3} \sin \theta\right) \cos \phi\right|
$$

where $H_{1}$ is the same as given in eqn (37). Hence there are two parameters $k_{2}$ and $k_{3}$ in this problem. However, this is a one-parameter problem since either $k_{2}$ or $k_{3}$ can be used as a scanning parameter. If we choose $k_{2}$ to be the scanning parameter, the full set of exact state equations $(10)$ can be integrated. It is necessary to use the explicit law (39) with a guessed value of $k_{3}$ and a bounded value of bank angle $\sigma_{\text {mik }}=85^{\circ}$. The initial state to be used is $(25)$ with $v_{1}=0.99$. At $v_{1}=0.001$, the transversality condition $p_{\omega_{1}}=0$ is used for the adjustment of the unknown parameter $k_{3}$.

To give both the scanning parameter and the unknown arbitrary parameter a physical meaning, the rotated coordinate system can also be used here. Here again wo have $\omega^{\prime}$, as the scanning parameter. The control law (35).

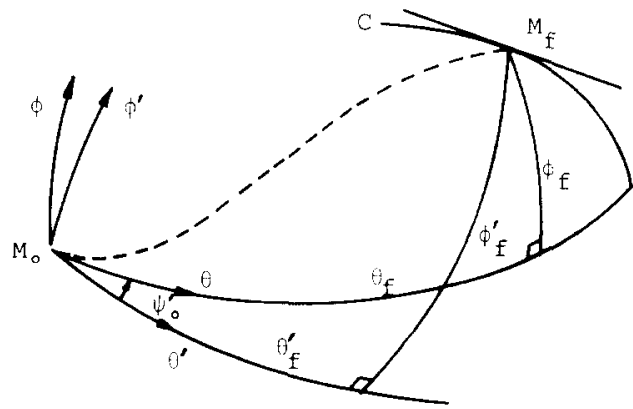

Fig. 3. Rotation of spherical coordinates 


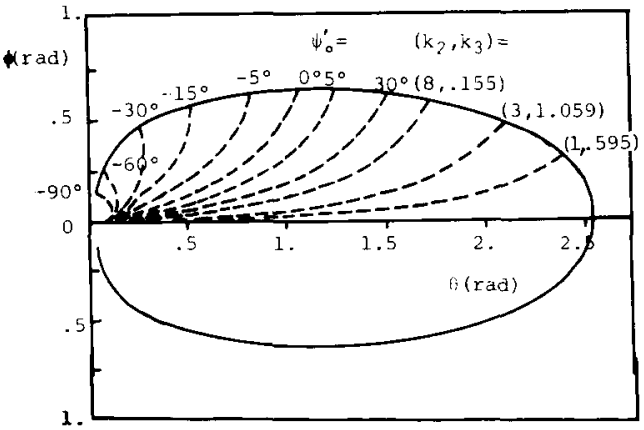

Fig. 4. Footprint without any constraint.

with the prime notation on $\theta . \phi$, and $\psi$, now becomes

$\tan \sigma=\frac{(1-v)}{v}$

$$
\frac{\cos \phi^{\prime} \sin \left(\theta_{f}^{\prime}-\theta^{\prime}\right)}{\cos \left(\theta_{f}^{\prime}-\theta^{\prime}\right) \sin \psi^{\prime}-\cos \psi^{\prime} \sin \phi^{\prime} \sin \left(\theta_{f}^{\prime}-\theta^{\prime}\right)} \text {. }
$$

The unknown parameter to be found is now $\theta_{f}^{\prime}$. Of course, the formulas to obtain $\left(\theta_{f}, \phi_{f}\right)$ from $\left(\theta_{f}^{\prime}, \phi_{f}^{\prime}\right)$ are the same as in (38). The footprint for $E^{*}=2$ is shown in Fig. 4, with the projections of the reentry trajectories on the
Earth's surface plotted in dashed lines. The variations of the dimensionless altitude, velocity ratio, flight path angle, and bank angle for the reentry tiajectories are plotted in Fig. 5. As has been shown in the previous section (Fig. 2), both the deceleration and the heating rate pass through several maxima. The first maxima are the highest. In Fig. 6, the highest decelerations encountered on all of the reentry trajectories leading to the footprint are plotted as a function of the longitudinal range $\theta_{f}$. It can be seen that to the left half of the footprint the highest decelerations are so large that it is necessary to impose a deccleration constraint on the reentry trajectories. Also shown in Fig. 6 are the highest heating rates encountered. We will also investigate reentry trajectories with heating rate constraint in the next section.

The full set of state equations are integrated using the optimal control law (40) deduced from the equilibrium glide condition. However, only the final speed can be prescribed since there is only one parameter. But it has been found that for all the trajectories, the final altitudes are uniformly close to the value $Z_{j}=30$. For most reentry vehicles. this represents a reasonably low altitude. Another thing to be mentioned is that, for $E^{*}=2$ with $\psi_{0}^{\prime}>30^{\circ}, \phi^{\prime}$ may increase to $90^{\circ}$ and cause a singularity to occur. Hence for that portion of the footprint beyond $\psi_{1}^{\prime}>30^{\circ}$, we should use eqn (39) to solve for the reentry trajectories and to complete the whole footprint.

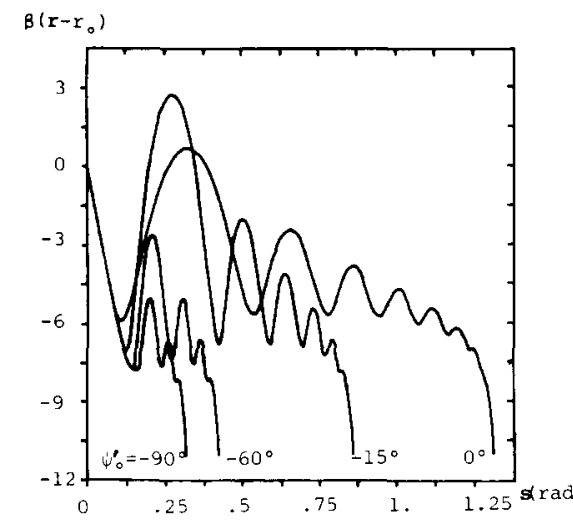

(a)

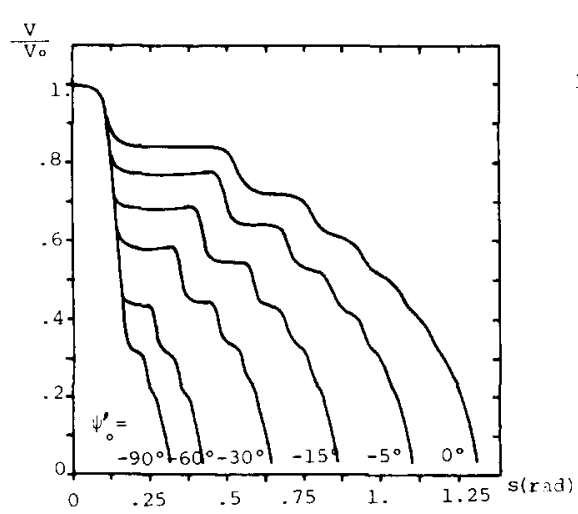

(b)

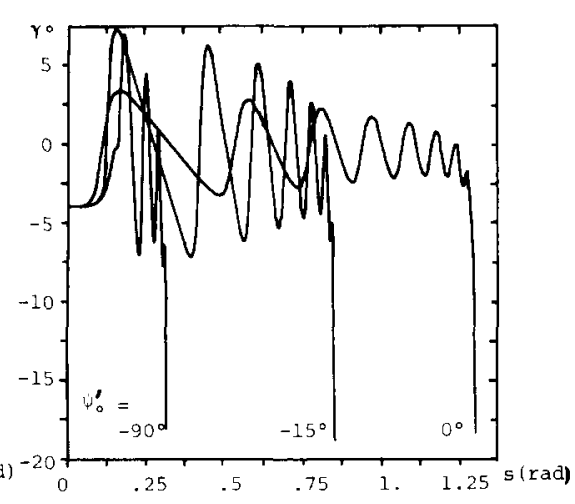

(c)

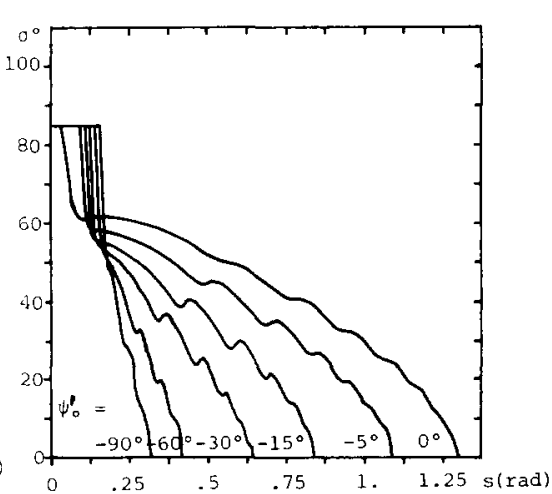

(d)

Fig. 5. Variations of (a) dimensionless altitude, (b) velocity ratio, (c) flight path angle, and (d) bank angle along unconstrained trajectories. 


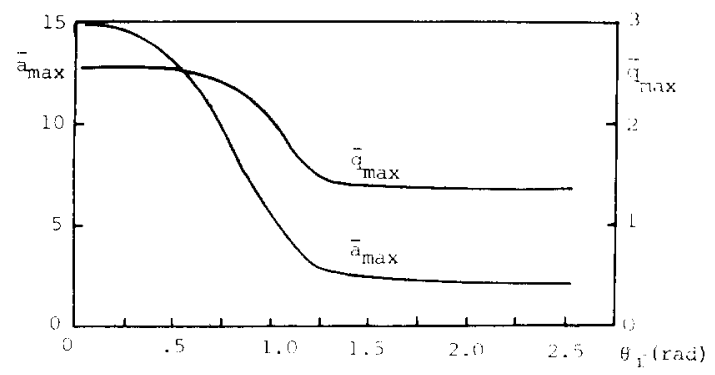

Fig. 6. $\bar{a}_{\text {max }}$ and $\bar{q}_{\text {mas }}$ along the unconstrained footprint.

\section{FOOTPRINTS WITH DECEIERATION ANI) HEATING; CONSTRAINTS}

\subsection{Footprint with a deceleration constraint}

As before, we shall use the equilibrium glide assumption and the technique of coordinate system rotation in this section. We shall use $\psi_{\prime \prime}^{\prime}=-90^{\circ},-60^{\circ},-30^{\circ}$. $-15^{\circ} .-5^{\circ} .0^{\circ} .5^{\circ}$ and $30^{\circ}$ to solve for the reentry trajectories and to generate most of the footprint with a deceleration constraint. The remaining portion of the footprint corresponding to $w_{\prime \prime}^{\prime}=30^{\circ}-90^{\circ}$ will be generated from the trajectories found by using eqn (39). The trajectory with $\omega_{,}^{\prime}=0^{\circ}$ is the one with global maximum cross range.

As shown in Fig. 5, the planar tlight trajectory which leads to the right end point of the footprint has the smallest first peak in deceleration. This trajectory gives the maximum longitudinal range, and has a first peak in deceleration of $\bar{a}_{t, 1}=2.12$. For planar flight under the equilibrium glide assumption, this trajectory is obtained by using the lift control $\lambda=1$ and the bank control $\sigma=0$. Hence it is always possible to control the deceleration on any trajectory down to the value $\bar{a}_{\text {mal }}=2.12$. This is of course at the expense of the reachable domain. Actually it is possible to further lower the peak deceleration in planar flight by lift modulation. but this problem will not be discussed here. We shall set the deceleration constraint values at $\bar{a}_{\text {tais }}=3.0$ and 2.12 . respectively. to generate the footprints and to investigate the reduction of the reachable domain due to the deceleration constraint.

To control the peak deceleration on a certain reentry trajectory, if the flight is required to stay at a certain $\bar{a}_{m a t}$ value, then we have

$$
k Z 1=\bar{a}_{11 \ldots \downarrow}
$$

By taking the derivative of this equation and making use of the exact state equations $(10)$, we have

$$
\frac{\left(1+\lambda^{2}\right)}{E^{*}} \bar{a}_{\max }=-\left[2+\left(k^{2}-1\right) w\right] \sin \gamma .
$$

Since the flight is effected at maximum lift-to-drag ratio, $\lambda=1$, using this value in eqn (42) gives the condition for entering the boundary $\bar{a}_{\max }$. Therefore, in order to reach the first peak in deceleration at the prescribed value $\bar{a}_{\text {max }}$, a constant $\theta_{i 1}^{\prime}$ must be selected for the parameter $\theta_{i}^{\prime}$ in eqn (40) such that eqns (41) and (42) are simulta- neously satisfied. Then by taking the derivative of eqn (42), the bank control appears explicitly and is found to be given by

$$
\begin{aligned}
& \bar{a}_{n \ldots} \cdot \cos (5)= \\
& \frac{\left.1\left(k^{2}-1\right)^{2}+\left(k^{2}-3\right) k+2\right] \cos ^{2} \gamma-k^{2}\left(k^{2}-1\right) 1^{2}}{[2+(k-1) \cdots \cos \gamma} .
\end{aligned}
$$

This is the optimal bank control for the flight to stay on the boundary $\bar{a}_{\text {ma: }}$. Then what we have to do next is to find the point for leaving the boundary. Since the bank control must be continuous at the point of leaving the boundary, we can combine the two eqns (40) and (43) to eliminate the bank angle and solve for the arbitrary parameter $\theta_{i}^{\prime}$. Here at the point of leaving the boundary it will be denoted by $\theta_{f}^{\prime}$ and the result is

$$
\begin{aligned}
\theta_{i=}^{\prime} & =\theta^{\prime}+\tan { }^{\prime} \\
& \times\left[\frac{v^{\prime} \sin \psi^{\prime} \tan \sigma}{(1-v) \cos \phi^{\prime}+v \sin \phi^{\prime} \cos \psi^{\prime} \tan \sigma}\right]
\end{aligned}
$$

where $\sigma$ is obtained from eqn (43). After leaving the boundary, eqn $(40)$ will be applied for the remaining flight of the trajectory. Finally when the integration of the trajectory is ended by the prescribed final value $r_{1}=0.001$, the final value $\theta_{i}^{\prime}$ is used to adjust the pat rameter $\theta_{2}$. In numerical integration. what we actually do is to adjust the instant of leaving the boundary. and check at the final instant to determine if $\theta_{f}^{\prime}$ and $\theta_{;}^{\prime}$ anc equal.

In summary, for a certain value of the scanning pa rameter $\psi_{\%}^{\prime}$. we start the integration of eqns $(10)$ with the initial condition (25) except that $r=0.99$. We use eqn $(40)$ with an initial guess of $\theta_{f}^{\prime}$, denoted by $\theta_{11}^{\prime}$, an bank control. The condition (42) is used to adjust $H_{1}^{\prime}$. Then on the prescribed $\bar{a}_{13: 3}$ boundary. the integration keeps on going by using eqn (43) as bank control so that the flight stays on the boundary. At a certain point the flight leaves the boundary and we continue the integration by using eyn (40) with the parameter computed from eqn (44). At the final instant $b_{i}=0.001$, the condition $\theta_{1}^{\prime} \cdots$ used to check the leaving point.

For the two deceleration constraint values $\bar{a}_{t \mid,}, \quad \cdots .0$ and 2.12, the above scheme is valid for us: $-90^{\circ} .-60^{\circ} .-30^{\circ}$. and $-15^{\circ}$. For $\omega^{\prime}--5^{\circ} .0^{\circ} .5^{\circ}$ $30^{\circ}$ and beyond, the flight does not have to stay on the boundary. In this case, the point of entering and the point of leaving the boundary coincide. This is because another value $\theta_{t}^{\prime}$ can be selected for the integration such that the prescribed final speed $1 ;, 0.001$ is identically satisfied at $\theta_{j}^{\prime}=\theta_{t_{2}^{\prime}}$. Modulated bank is used through eqn (40).

The footprint without any constraint, the footprint with a constraint of $\bar{a}_{t w 1,}=3.0$, and the footprint with a constraint of $\bar{a}_{\text {thls }}=2.12$ are presented in Fig. 7. The var iations of the dimensionless altitude. velocity ratio. hight path angle and bank angle for $\vec{a}_{11,4}-2.12$ are shown in Fig. 8. Here the dimensionless attitude $\Delta \bar{h}$ is defined to 


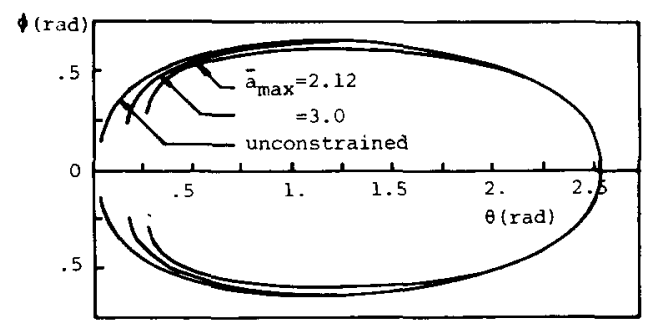

Fig. 7. Footprints with and without constraints on $\bar{a}_{\max }$

be the actual altitude drop divided by $1 / \beta$. Hence we have

$$
\Delta \bar{h}=\ln \left(Z_{p} / Z_{f}\right) .
$$

The velocity ratio is defined to be the ratio between the actual speed and the initial speed and can be obtained by simply taking the square root of $v$.

\subsection{Footprint with a heating constraint}

We shall set the dimensionless heating rate constraint values at $\bar{q}_{\max }=1.5$ and 1.36 to generate the footprints.
The value $\bar{q}_{\max }=1.36$ is reached by the first peak of heating rate on the planar reentry trajectory. Hence it is always possible to control the heating rate on any reentry trajectory down to this value.

To enter the boundary of a controlled peak heating rate $\bar{q}_{\max }$, we need

$$
\left(k Z v^{3}\right)^{1 / 2}=\bar{q}_{\max }
$$

and

$$
\frac{3\left(1+\lambda^{2}\right)}{E^{*}} \bar{q}_{\operatorname{mux}}^{2}=-v^{2}\left[6+\left(k^{2}-3\right) v\right] \sin \gamma
$$

where the entering condition (47) is obtained by taking the derivative of eqn (46). From the initial condition (25) with $v_{o}=0.99$ we start the integration of the exact state equations (10). We use eqn (40) with an initial guess of $\theta_{f}^{\prime}$, denoted by $\theta_{f 1}^{\prime}$, as bank control. The condition (47) is used to adjust $\theta_{i 1}^{\prime}$. Then on the prescribed $\bar{q}_{\max }$ boundary, the control law for the bank angle on the boundary can be obtained by taking the derivative of eqn (47)

$$
\bar{q}_{\max }^{2} \cos \sigma=\frac{v^{2}\left\{\left[\left(k^{2}-1\right)\left(k^{2}-3\right) v^{2}+\left(5 k^{2}-9\right) v+6\right] \cos ^{2} \gamma-k^{2} v\left[4+\left(k^{2}-3\right) v\right]\right\}}{\left[6+\left(k^{2}-3\right) v\right] \cos \gamma} .
$$

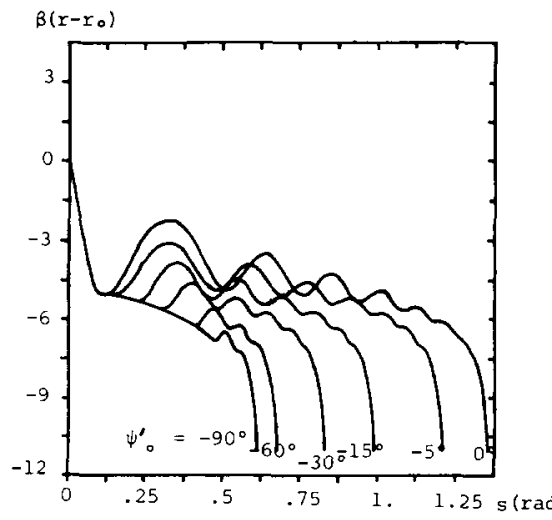

(a)

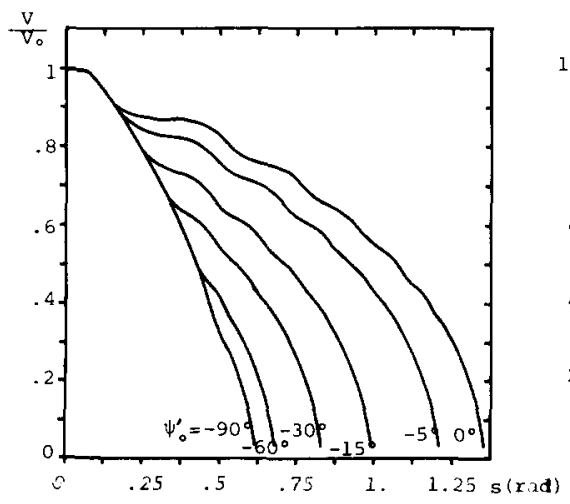

(b)

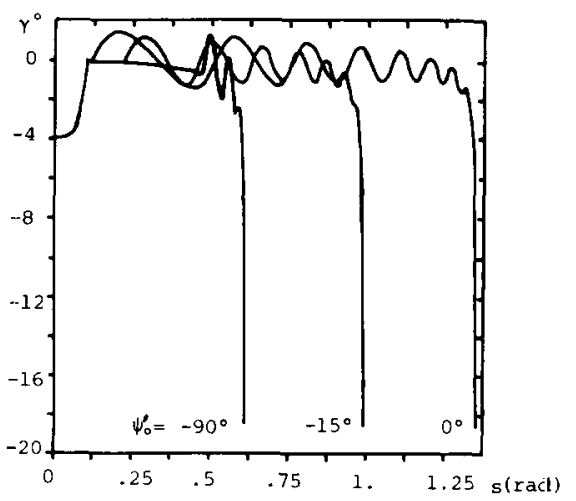

(c)

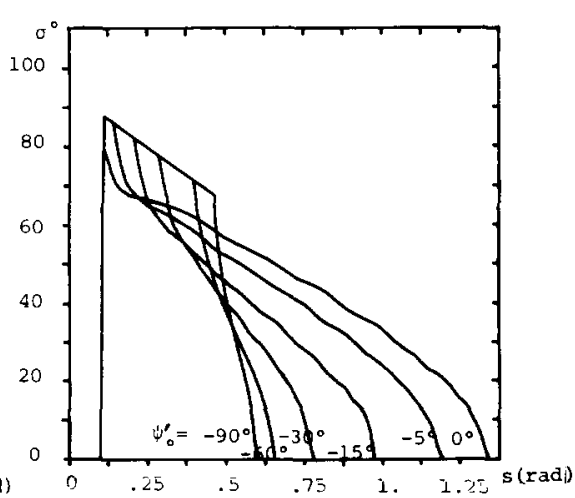

(d)

Fig. 8. Variations of (a) dimensionless altitude, (b) velocity ratio, (c) flight path angle, and (d) bank angle along trajectories with a constraint of $\bar{a}_{\max }=2.12$. 
$\phi(r a d)$

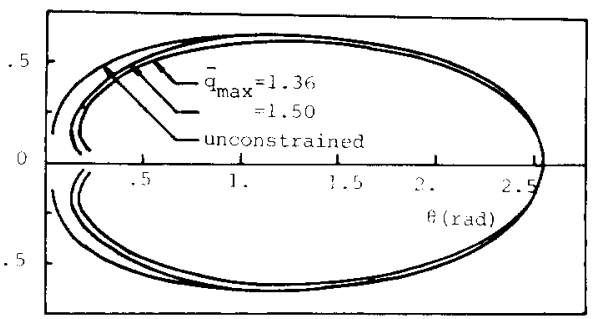

Fig. 9. Footprints with and without constraints on $\bar{q}_{\text {mux }}$.

Finally at the point of leaving the boundary, the arbitrary parameter, also denoted by $\theta_{t 2}^{\prime}$, is computed from eqn (44) with the bank angle $\sigma$ obtained from eqn (48). This parameter is to be used in eqn (40) for the modulated bank control after leaving the boundary. When the prescribed final speed $v_{f}=0.001$ is reached, the condition $\theta_{f}^{\prime}=\theta_{f 2}^{\prime}$ is used to adjust the leaving point.

Again, for the two prescribed heating constraint values $\bar{q}_{\text {max }}=1.5$ and 1.36 , it is not necessary to maintain the trajectory at a constant heating rate that is on the constraint boundary, for $\psi_{1 \prime}^{\prime}=0^{\circ}, 5^{\circ}, 30^{\circ}$ and beyond. As has been found in previously published literature[1], it is possible to find a new constant $\theta_{f 2}^{\prime}$ for modulated bank control satisfying the transversality conditions $p_{\omega_{f}}=0$ and $p_{\mathrm{b}_{f}}=1$, or simply $\sigma\left(\theta_{i_{2}}^{\prime}\right)=0$, at a prescribed $v_{f}$ while the heating rate remains less than $\bar{q}_{\text {mar. }}$.

In Fig. 9, the footprints without any constraint, with a constraint of $\bar{q}_{\text {max }}=1.5$, and with a constraint of $\bar{q}_{\text {max }}=1.36$, respectively, are plotted. The variations of the dimensionless altitude, dimensionless speed. flight path angle, and bank angle for $\bar{q}_{\text {mil }}=1.36$ are shown in Fig. 10.

\subsection{Discussions}

In Fig. 11, the maximum heating rates encountered on the trajectories without any constraint and with a constraint of $\bar{a}_{\text {mix }}=2.12$ are plotted along the footprints. Also plotted are the maximum decelerations cncountered on the trajectories without any constraint and with a constraint of $\bar{q}_{\text {max }}=1.36$ imposed along the footprints. It is seen that we have dual reduction. This means that on the deceleration constrained reentry trajectory the heating rato is also reduced, and vice versa except on the trajectories for very small values of $\theta_{f}$. The deceleration control also brings the heating rate to an acceptable level. The penalties on the reachable domain are shown in Figs. 7 and 9.

Mathematically. immediately after entering the constrained boundary, an estimated value of $\theta$ is selected to compute $\sigma$ by using eqn (40). The result is compared with the value obtained from eqn (43) or (48). If the modulated $\sigma$ is higher, the flight is required to stay on the boundary until the two values are equal. Then the flight switches to modulated bank until the final value $\sigma_{t}=0$ at the prescribed final speed. At the point of entering the constrained boundary the bank angle displays

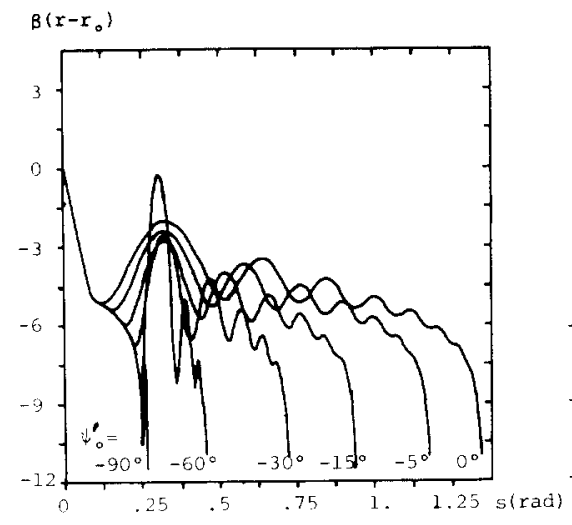

(a)

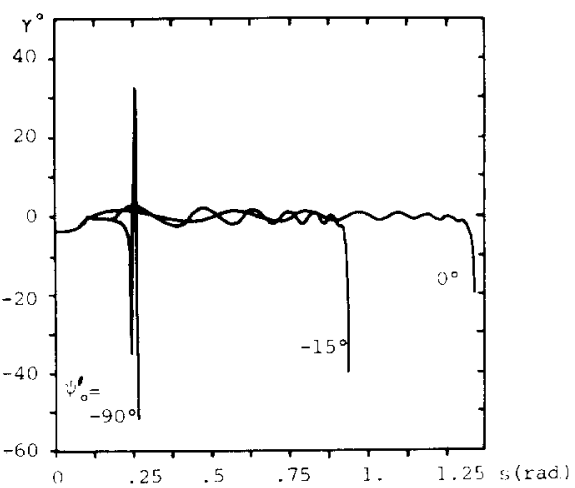

(c)

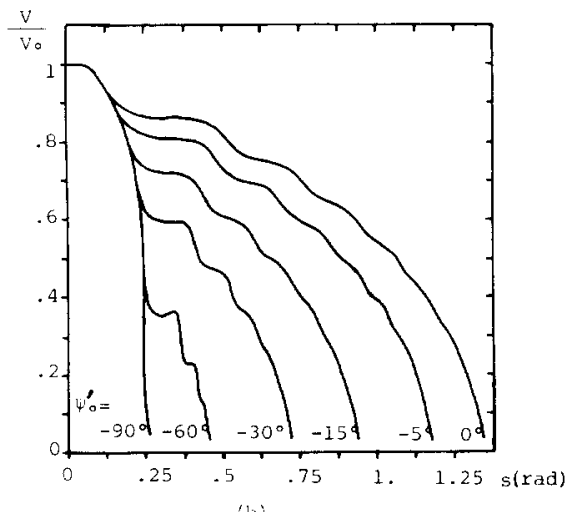

(1)

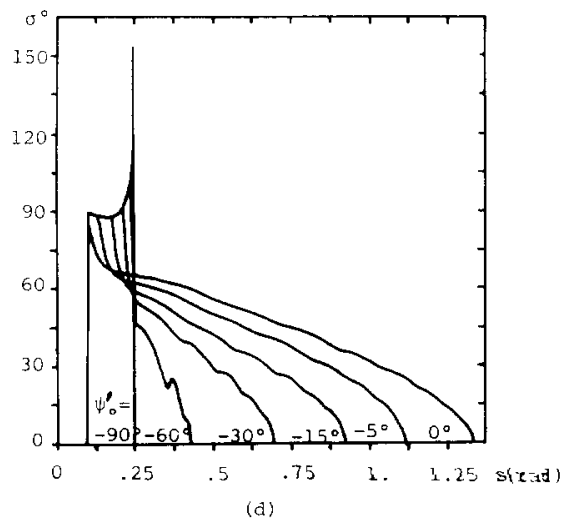

Fig. 10. Variations of (a) dimensionless altitude, (b) velocity ratio, (c) flight path angle, and (d) bank angle along trajectories with a constraint of $\bar{q}_{\max }=1.36$ 


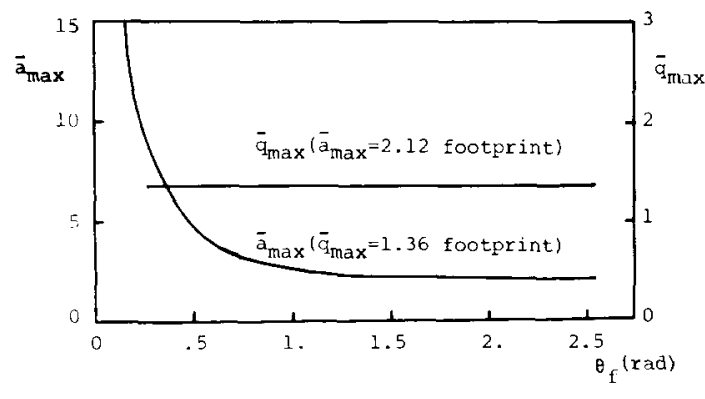

Fig. 11. $\bar{q}_{\max }$ and $\bar{a}_{\max }$ along the constrained footprints.

a discontinuity. The reason is that its explicit law is obtained by taking the second derivative of the constraining relation, either eqn (41) or (46), in the case where the flight is required to stay on the boundary. Otherwise, it is computed by using the same equation, namely (40), but with a different parameter value $\theta_{f 2}^{\prime}$ in the case the flight is not required to stay on the boundary.

\section{VARIATION OF BANK ANGLE ON THE BOUNDARY}

It is of interest to analyze the variation of $\sigma$ on the constrained boundary. For controlled deceleration at $\bar{a}_{\max }, \cos \sigma$ can be expressed explicitly as function of $v$ alone by using eqn (42) to calculate $\gamma$ and substituting into eqn (43). If the simplification $\cos \gamma \approx 1$ is made, we obtain directly from (43)

$$
\bar{a}_{\max } \cos \sigma=1-v .
$$

This is the same as using the equilibrium glide condition (33) as the control law. It appears that when $\vec{a}_{\max }$ is small, the bank angle is small. Since this bank angle is used to maintain constant $\bar{a}_{\max }$, it is substituted for the modulated bank angle if this angle is higher. Also, since the optimal final value of $\sigma$ is $\sigma_{f}=0$, the trajectory will leave the constrained boundary at a certain point before the end.

Similarly, using the approximation $\cos \gamma \approx 1$ in eqn (48), we have the simple formula

$$
\vec{q}_{\max }^{2} \cos \sigma=v^{2}(1-v),
$$

which can also be obtained by using the constraining relation (46) in the equilibrium glide condition (33). The same remarks as for the case of flight on the boundary $\bar{a}_{\max }$ may be made. In addition, from eqn (50), we see that $\sigma$ passes through a minimum at the value $v=2 / 3$. This gives

$$
\sigma_{\min }=\cos ^{-1}\left(\frac{4}{27 \bar{q}_{\text {max }}^{2}}\right) .
$$

This minimum value is low for a low value of $\bar{q}_{\text {max }}$ and occurs at the speed ratio $V / V_{0}=0.816$. After passage through this point the bank angle increases, ultimately switches to modulated bank control and decreases to the final value $\boldsymbol{\sigma}_{f}=0$.

\section{CONCLUSIONS}

This paper gives a general assessment of the footprint as a function of various deceleration and heating con- straints. With the dimensionless equations of motion, it is possible to specify just the maximum lift-to-drag ratio $(L / D)_{\max }$ as the sole physical characteristics of the vehicle. Hence the analysis applies to a whole class of vehicles having the same value for $(L / D)_{\max }$. The exact optimal trajectory for global maximum cross range is found first. This is a three-parameter problem. We have used the classical integrals of the motion, and at the same time transformed the adjoint variables into physical variables. The integrals of the motion allow a reduction of the order of the canonical system. The transformation of the adjoint variables into physical variables makes the initial guess of unknown constants a much less arbitrary task. The equilibrium glide condition is then introduced to reduce the problem to a one-parameter problem. The trajectory for global maximum cross range is found under the assumption of equilibrium glide and it is concluded that this is a very good suboptimal solution. The footprint without any constraint, the footprint with a deceleration constraint, and the footprint with a heating rate constraint are generated to show the reduction of the reachable domain due to different imposed constraints. The equilibrium glide assumption reduced much of the numerical computation load. Furthermore, the technique of rotating the coordinate system on the spherical earth reduces the computation of the footprints to a swept operation through a scanning heading angle. The reduction in the size of the reachable domain is substantial only on the left half portion of the footprint. It is seen that on the deceleration constrained reentry trajectory the heating rate is also reduced, and vice versa. Also, deceleration control generally brings the heating rate to an acceptable level. For the reentry trajectories with small prescribed longitudinal ranges, the flight has to stay on the constraint boundary upon entering the specified constrained value. The smaller the longitudinal range is, the longer the flight stays on the boundary before leaving it. The variation of the bank angle on the constraint boundary is discussed in detail.

\section{REFERENCES}

1. L. M. Shkadov, R. S. Bukhanova, V. F. Illarionov and V. P. Plokhikh, Mechanics of optimum three-dimensional motion of aircraft in the atmosphere. NASA TT F-777 (1975)

2. D. G. Hull and J. L. Speyer, Optimal reentry and planechange trajectories. J. Astronaut. Sci. 117-130 (1982).

3. J. S. Chern and N. X. Vinh, Optimum reentry trajectories of a lifting vehicle. NASA CR-3236 (1980).

4. N. X. Vinh, Optimal Trajectories in Atmospheric Flight. Elsevier, Amsterdam (1981).

5. J. S. Chern, C. Y. Yang, N. X. Vinh and G. R. Hwang, Optimal three-dimensional reentry trajectories subject to deceleration and heating constraints. IAF-82-309, 33rd Congr. IAF, Paris (1982)

6. A. Miele, Flight Mechanics, Vol. I. Theory of Flight Paths Addison-Wesley, Reading, MA (1962).

7. N. X. Vinh, A. Busemann and R. D. Culp, Hypersonic and Planetary Entry Flight Mechanics. University of Michigan Press, Ann Arbor, MI (1980).

8. L. Lees, Laminar heat transfer over blunt-nosed bodies at hypersonic flight speeds. Jet Propulsion 26, 259-269 (1956).

9. J. Fave, Approche analytique du problème du domaine accessible à un planeur orbital. La Recherche Aérospatiale $\mathbf{1 2 4}$ $3-11(1968)$ 